Proc. Indian Acad. Sci. (Chem. Sci.), Vol. 105, No. 6, December 1993, pp. 637-649.

(C) Printed in India.

\title{
Exciplex mechanism of fluorescence quenching in polar media
}

\author{
MICHAEL G KUZMIN*, NIKITA A SADOVSKII, \\ JULIA WEINSTEIN and OLEG KUTSENOK \\ Department of Chemistry, Moscow University, Moscow 117234, Russia
}

\begin{abstract}
The formation of exciplexes (non-emitting or poorly emitting) is suggested as one of the causes for deviations of experimental data on fluorescence quenching in polar solvents from the classical model of excited-state electron transfer yielding radical ion pairs. Several evidences for the formation of such exciplexes were found for fluorescence quenching of aromatic compounds by weak electron donors and acceptors. For cyano-substituted anthracenes exciplex emission can be observed in the presence of quenchers even in polar solvents. In other systems, indirect evidences of exciplex formation were observed: nonlinear dependence of the inverse value of excited pyrene lifetime os the concentration of the quencher; very small and, in some cases, even negative experimental activation energies of pyrene fluorescence quenching, which are much less than activation energies, calculated from the experimental values of the quenching rate constants etc.

The proposed model explains the difference between theoretical and experimental dependencies of $\log k_{\mathrm{Q}}$ vs. Gibbs energy of electron transfer $\Delta G_{\mathrm{ET}}$ and other experimental features known for fluorescence quenching by electron donors and acceptors. This model states that the exciplex is in equilibrium with the encounter complex and apparent quenching rate constants are controlled by two main factors - the lifetime of the exciplex and the enthalpy of its formation. Experimentally observed dependence of apparent quenching rate constant on $\Delta G_{E T}$ is caused by the dependence of the exciplex formation enthalpy on $\Delta G_{E T}$, which is quite different from the dependence of electron transfer activation energy on $\Delta G_{E T}$ predicted by the theoretical models. Simulations of the dependencies of $\log k_{\mathbf{Q}}$ vs. $\Delta G_{\mathrm{ET}}$ according to the exciplex formation model confirms its agreement with the experimental data.

Electronic structure of the exciplex involved may be close to contact radical-ion pair only at $\Delta G_{E \mathrm{~T}}<0$, when the rate of quenching is limited mainly by the diffusion, but for $\Delta G_{\mathrm{ET}}>0$, the structure of the exciplex should be much less polar.
\end{abstract}

Keywords. Fluorescence quenching; polar media; exciplex mechanisms; excited-state electron transfer.

\section{Introduction}

Electron transfer photoreactions are very important in chemistry and biology. Excitedstate electron transfer is supposed to be a general mechanism oi fluorescence quenching in the absence of energy transfer and heavy atom effects. It is necessary to know the real mechanism of this process prior to the discussion of various theoretical models of electron transfer.

The classical kinetic scheme proposed by Rehm and Weller $(1969,1970)$ assumes

\footnotetext{
* For correspondence
} 
that the electron transfer step yields contact radical-ion pairs which dissociate very fast in polar solvents $\left(k_{30}\right.$ is about $\left.10^{11} \mathrm{~s}^{-1}\right)$ :

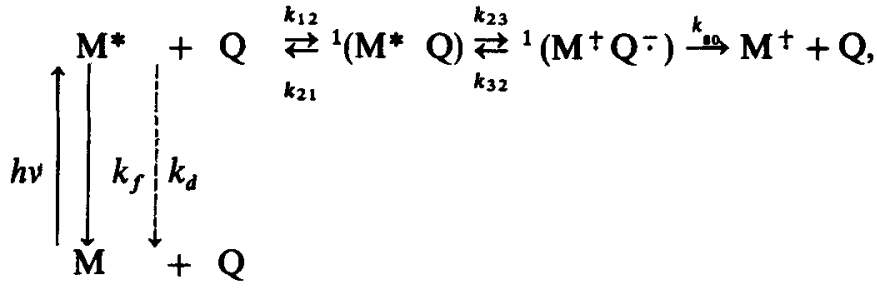

$$
\begin{aligned}
& k_{\mathrm{Q}}=k_{12} /\left[1+\left(1+k_{32} / k_{30}\right) k_{21} / k_{23}\right] \text {, } \\
& \varphi_{0} / \varphi=\tau_{0} / \tau=1+k_{\mathrm{Q}} \tau_{0}[\mathrm{Q}] \text {, }
\end{aligned}
$$

where $\varphi_{0}, \varphi$ and $\tau_{0}, \tau$ are fluorescence quantum yields and lifetimes in the absence and in the presence of a quencher $\mathrm{Q}$, respectively $\left(1 / \tau_{0}=k_{f}+k_{d}\right)$.

A number of experimental investigations (Fox and Chanon 1988) showed the existence of the typical dependence of the quenching rate constants $k_{\mathrm{Q}}$ in polar solvents on the Gibbs energy of electron transfer $\Delta G_{\mathrm{ET}}$ which has a diffusion limit for $\Delta G_{\mathrm{ET}} \ll 0$ and kinetic limit for $\Delta G_{\mathrm{ET}} \geqslant 0$. This experimental dependence is in good agreement with the kinetic scheme (1), if one assumes that activation energy of an electron transfer step depends on $\Delta G_{\mathrm{ET}}$ according to the empirical Weller's equation (Rehm and Weller 1969, 1970),

$$
\Delta G_{23}^{\neq}=\Delta G_{\mathrm{ET}} / 2+\left[\left(\Delta G_{\mathrm{ET}} / 2\right)^{2}+\left(\Delta G_{0}^{\neq}\right)^{2}\right]^{1 / 2},
$$

or theoretical Marcus' equation (Marcus 1956).

$$
\Delta G_{23}^{\neq}=\Delta G_{0}^{\neq}\left(1+\Delta G_{E T} / a \Delta G_{0}^{\neq}\right)^{2} .
$$

But numerous experimental data obtained in recent years for various systems (Hishimura et al 1977, Baggott and Pilling 1983, Kitamura et al 1987, Avila et al 1991, Carrera et al 1991, Neumann and Pastre 1991 etc.) have shown that such correlation is qualitative rather than quantitative. Wide scatter is observed for $k_{\mathrm{Q}}$ at $\Delta G_{\mathrm{ET}} \geqslant 0$. Even values of $k_{Q}$ greater than thermodynamically permitted for electron transfer were observed.

To find out the origin of these deviations we investigated fluorescence quenching kinetics and determined the activation energies of quenching from the temperature dependence of the apparent quenching constants in several systems. Fluorescence quenching of pyrene and 9-cyanoanthracene by some weak electron donors and acceptors was studied in acetonitrile $\left(\Delta G_{\mathrm{ET}} \approx 0\right)$ and in other solvents (Kuzmin and Soboleva 1986; Kuzmin et al 1992). We found several evidences that fluorescence quenching even in polar solvents can proceed by the formation of exciplexes rather than by direct electron transfer mechanism:

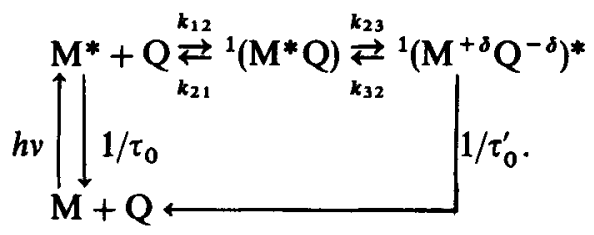


In the case of equilibrium between the excited molecules and the exciplex the value of the apparent quenching rate constant is controlled by the lifetime of the exciplex and equilibrium constant of its formation rather than by the rate constant of electron transfer.

Exciplexes are well known to be formed in electron transfer photoreactions in non-polar media (Gordon and Ware 1975). But in polar media no exciplex emission was observed in most cases and electron-transfer reactions are usually assumed to yield radical-ion pairs which dissociate very fast $\left(\sim 10^{-10}-10^{-11} \mathrm{~s}\right)$ and produce free radical-ions.

Kinetics of exciplex formation in non-polar solvents were studied in various systems (Kuzmin and Soboleva 1986):

$$
\left.\stackrel{\mathrm{M}^{*}+\mathrm{Q} \underset{k-1}{\stackrel{k_{1}}{\rightleftarrows}}{ }^{1}\left(\mathrm{M}^{+\delta} \mathrm{Q}^{-\delta}\right)^{*}}{1 / \tau_{0}}\right|^{1 / \tau_{0}^{\prime} .}
$$

Fluorescence quenching follows the Stern-Volmer equation, but the observed Stern-Volmer quenching constant has completely different sense:

$$
\varphi_{0} / \varphi=1+K_{\mathrm{sV}}[\mathrm{Q}]=1+k_{1} \tau_{0}[\mathrm{Q}] /\left(1+k_{-1} \tau_{0}^{\prime}\right)
$$

The ratio of exciplex $\left(\varphi^{\prime}\right)$ and initial molecule $(\varphi)$ fluorescence quantum yields linearly depends on the quencher concentration:

$$
\varphi^{\prime} / \varphi=k_{f}^{\prime} k_{1} \tau_{0}[\mathrm{Q}] / k_{f}\left(1+k_{-1} \tau_{0}^{\prime}\right)
$$

Initial molecule fluorescence decay kinetics $(f(t))$ is biexponential and exciplex fluorescence kinetics $\left(f^{\prime}(t)\right)$ is the difference of the same exponents:

$$
\begin{aligned}
& f(t)=f_{0}\left[\alpha \exp \left(-t / \tau_{1}\right)+\exp \left(-t / \tau_{2}\right)\right], \\
& f^{\prime}(t)=f_{0}^{\prime}\left[\exp \left(-t / \tau_{2}\right)-\exp \left(-t / \tau_{1}\right)\right] .
\end{aligned}
$$

Dependence of the lifetimes of these exponents on the quencher concentration, in the general case, is nonlinear and can be expressed by the following expressions:

$$
\begin{aligned}
1 / \tau_{1,2}= & 1 / \tau_{0}+k_{1}[\mathrm{Q}]+1 / \tau_{0}^{\prime}+k_{-1} \\
& \pm\left[\left(1 / \tau_{0}+k_{1}[\mathrm{Q}]-1 / \tau_{0}^{\prime}-k_{-1}\right)^{2}+4 k_{1} k_{-1}[\mathrm{Q}]\right]^{1 / 2}, \\
\alpha= & \left(1 / \tau_{0}+k_{1}[\mathrm{Q}]-1 / \tau_{2}\right) /\left(1 / \tau_{1}-1 / \tau_{0}-k_{1}[\mathrm{Q}]\right) .
\end{aligned}
$$

In the case of reversible exciplex formation $\left(k_{-1} \gg 1 / \tau_{0}^{\prime}\right)$, at $\tau_{0} \gg \tau_{0}^{\prime}$, the dependence of $\tau_{0} / \tau_{2}$ on [Q] is sublinear ( $\tau_{1}$ is very short at $k_{1} \tau_{0}$ [Q] $\gg 1$ and may be imperceptible)

$$
\tau_{0} / \tau_{2} \approx\left(1+\left(\tau_{0} / \tau_{0}^{\prime}\right) K_{\mathrm{Ex}}[\mathrm{Q}]\right) /\left(1+K_{\mathrm{Ex}}[\mathrm{Q}]\right)
$$

$\left(K_{\mathrm{Ex}}=k_{23} / k_{32}\right.$ is the exciplex formation equilibrium constant), and has initial slope different from the Stern-Volmer constant for $\varphi_{0} / \varphi$ and reaches the limit equal to $\tau_{0} / \tau_{0}^{\prime}$. With the increase of concentration of the quencher, the observed lifetime of $\mathbf{M}^{*}$ (which is in equilibrium with the exciplex) falls to the limit determined by the exciplex 
lifetime. For $\tau_{0} \ll \tau_{0}^{\prime}$, the dependence of $1 / \tau_{2}$ on [Q] can even have a negative initial slope.

\section{Evidence for exciplex formation in polar solvents}

Fluorescence quenching of pyrene and 9-cyanoanthracene by some weak electron donors and acceptors in acetonitrile and other polar solvents follows the SternVolmer equation (figure 1)

$$
\varphi_{0} / \varphi=1+K_{\mathrm{sv}}[\mathrm{Q}] .
$$

A weak new emission band was observed for 9-cyanoanthracene in the presence of 1,6-dimethylnaphthalene (DMN) (figure 2) but no new emission bands were observed in the presence of the quenchers in most of the other systems investigated. This means that exciplexes have low emission rate constants or very short lifetimes. The apparent quenching rate constant according to scheme (6) can be expressed as

$$
k_{\mathrm{Q}}=K_{\mathrm{sv}} / \tau_{0}=k_{1} /\left(1+k_{-1} \tau_{0}^{\prime}\right) .
$$

The ratio of the emission quantum yields of 9-cyanoanthracene and its exciplex linearly depends on the concentration of DMN (figure 3) according to (9). Fluorescence decay kinetics of 9-cyanoanthracene in the presence of DMN is biexponential and fluorescence kinetics of its exciplex is the difference between the same exponents, [(10)-(11)].

Pyrene fluorescence decays monoexponentially (at least two orders of magnitude) in the absence and the presence of the quenchers but the lifetimes do not follow the

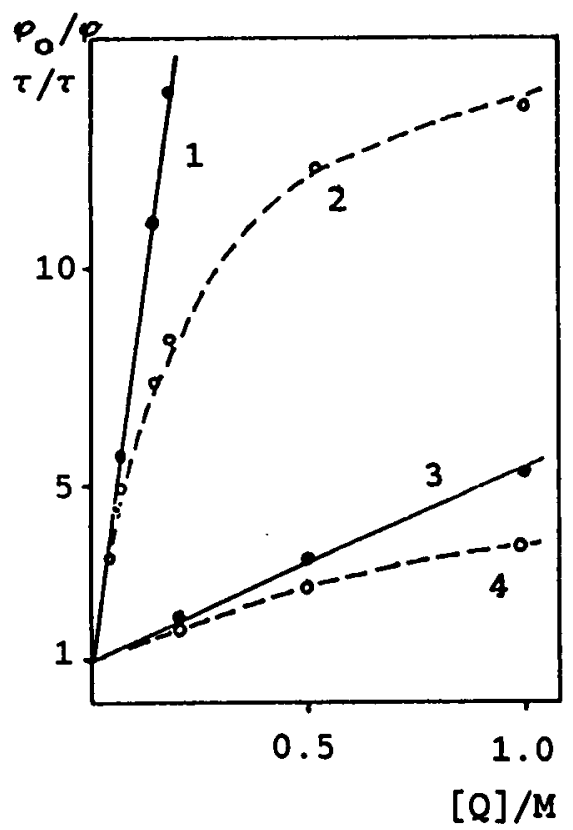

Figure 1. Plots of pyrene relative fluorescence quantum yields $\varphi_{0} / \varphi(1,3)$ and lifetimes $\tau_{0} / \tau$ $(2,4)$ vs. concentration of dibutylphthalate in acetonitrile $(1,2)$ and in butyronitrile $(3,4)$. 


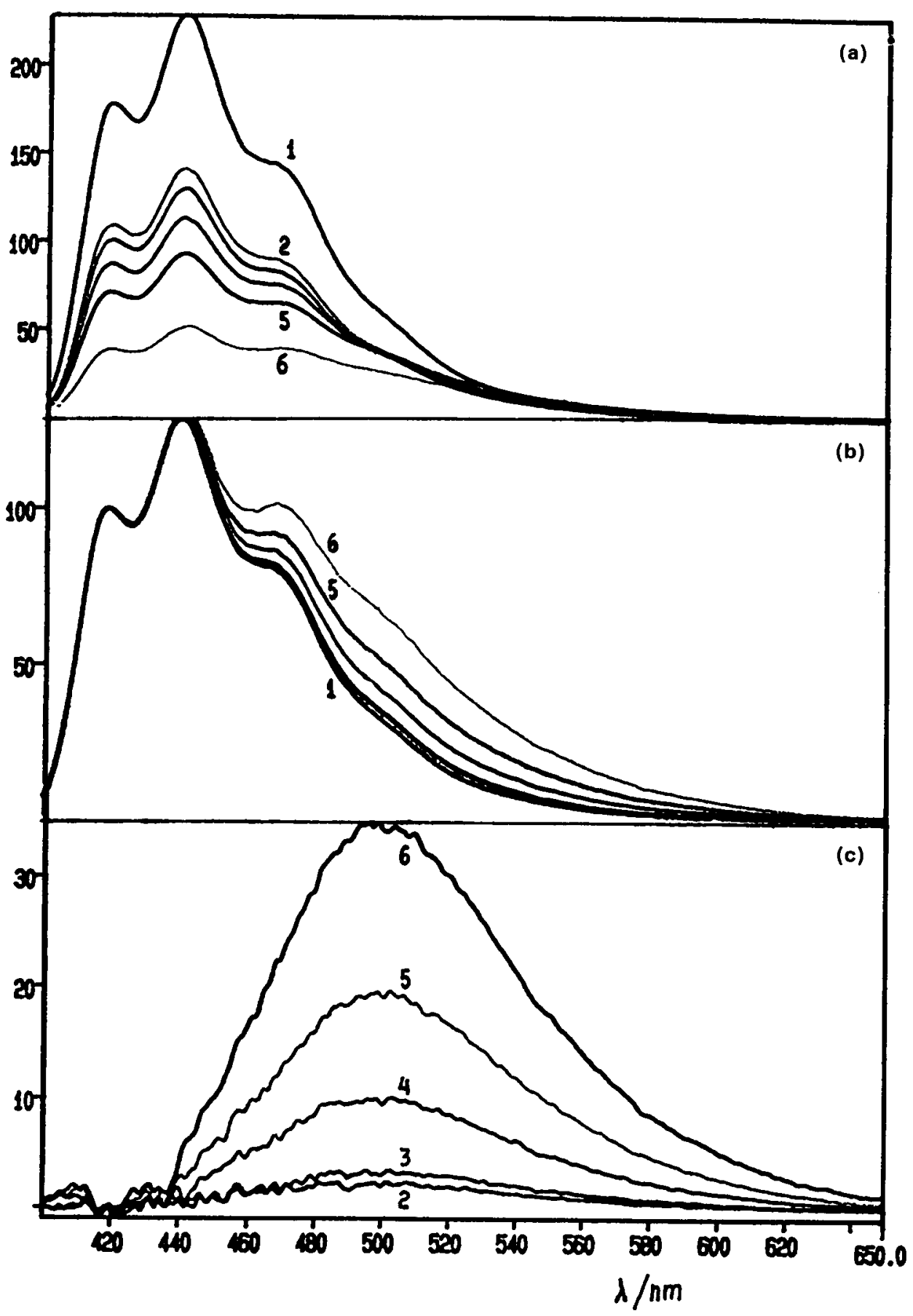

Figure 2. Uncorrected fluorescence spectra of 9-cyanoanthracene in the presence of various concentrations of 1,6-dimethylnaphthalene in acetonitrile (a) $(1-6: 0,2,4,9,20,40 \mathrm{mM}$ ), normalized fluorescence spectra (b) and exciplex emission spectra (extracted from overall spectra) (c). 


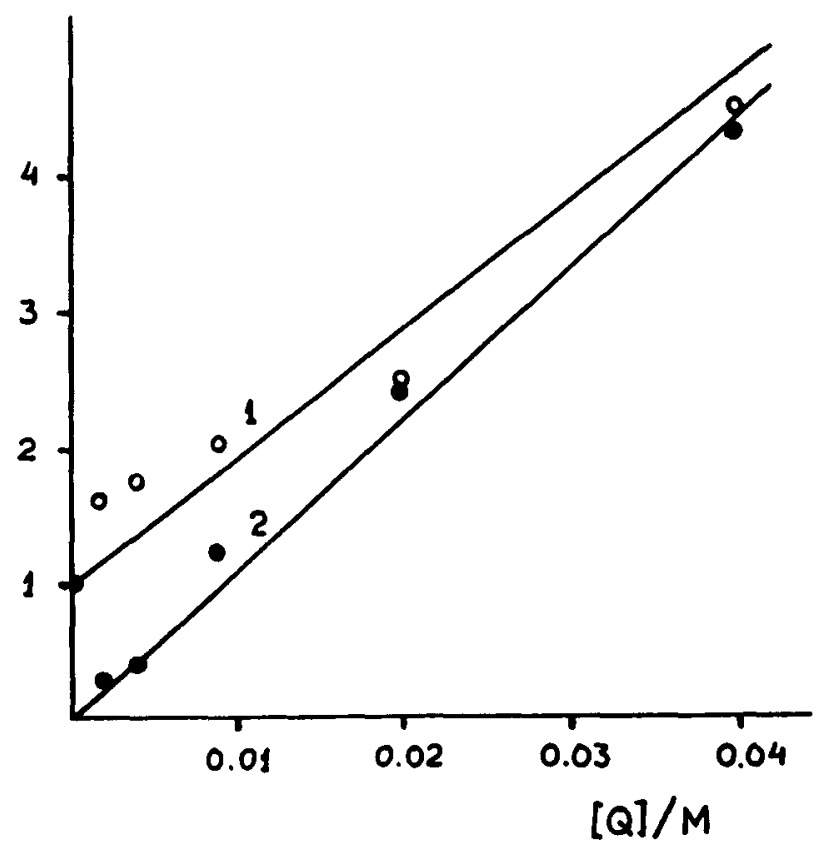

Figure 3. Plots of relative fluorescence quantum yields of 9-cyanoanthracene $\left(\varphi_{0} / \varphi, 1\right)$ and of the exciplex $\left(\varphi^{\prime} / \varphi, 2\right)$ vs. concentration of 1,6-dimethylnaphthalene in acetonitrile.

Stern-Volmer equation $-1 / \tau$ sublinearly depends on the quencher concentration (figure 1), which is typical for exciplex formation, (14). Plots of [Q]/( $\left.\tau_{0} / \tau-1\right)$ and $\varphi_{0} \tau / \varphi \tau_{0}$ vs. [Q] give $K_{\mathrm{Ex}}$ and $\tau_{0} / \tau_{0}^{\prime}$ :

$$
\begin{aligned}
{[\mathrm{Q}] /\left(\tau_{0} / \tau-1\right) } & =\left(1 / K_{\mathrm{Ex}}+[\mathrm{Q}]\right) /\left(\tau_{0} / \tau_{0}^{\prime}-1\right), \\
\varphi_{0} \tau / \varphi \tau_{0} & =1+K_{\mathrm{Ex}}[\mathrm{Q}] .
\end{aligned}
$$

The values of $K_{\mathrm{Ex}}$ and $\tau_{0}^{\prime}$ obtained are given in table 1 .

To confirm the nature of the nonlinear dependence of $1 / \tau$ on [Q] we studied this dependence in the presence of additional quenchers $\left(\mathrm{O}_{2}\right.$ and dimethylfumarate which quench both excited pyrene molecules and the exciplexes and change $\tau_{0}$ and $\tau_{0}^{\prime}$ ) and obtained the same values of $K_{\mathrm{Ex}}$. The observed difference in $\tau_{0}$ and $\tau_{0}^{\prime}$ show that both excited pyrene molecules and exciplex are quenched by oxygen and by dimethylfumarate with diffusion rate constants. It is important that exciplex lifetimes are relatively long - from 10 to $30 \mathrm{~ns}$. This means that the reason for the absence of exciplex emission for these systems is the very low value of the emission rate constant $\left(k_{f}^{\prime}<10^{6} \mathrm{~s}^{-1}\right)$.

Another proof of exciplex formation is the very low (and in some cases even negative) value of experimental activation energy of quenching, determined from the temperature dependence of the apparent quenching rate constant in the range $(-40)$ to $(+60)^{\circ} \mathrm{C}$. Apparent activation energy determined this way according to (16) is a sum of the exciplex formation enthalpy (negative) and its decay activation energy (small and positive). 
Table 1. Experimental data for pyrene and 9-cyanoanthracene. Stern-Volmer fluorescence quenching constants $K_{\mathrm{sv}}$, apparent quenching rate constants $k_{\mathrm{Q}}$ (at $298 \mathrm{~K}$ ), experimental activation energies $E_{\text {exp }}^{*}$ and exciplex formation equilibrium constants $K_{\mathrm{Ex}_{\mathrm{x}}}$ and lifetimes $\tau_{0}^{\prime}$.

\begin{tabular}{|c|c|c|c|c|c|c|}
\hline Quencher & Solvent & $\begin{array}{l}K_{\mathrm{sv}} \\
\left(\mathbf{M}^{-1}\right)\end{array}$ & $\begin{array}{l}k_{\mathrm{Q}} / 10^{7} \\
\left(\mathrm{M}^{-1} \mathrm{~s}^{-1}\right)\end{array}$ & $\begin{array}{c}E_{\text {exp }}^{*} \\
(\mathrm{~kJ} / \mathrm{mol})\end{array}$ & $\begin{array}{c}K_{\mathrm{Ex}} \\
\left(\mathrm{M}^{-1}\right)\end{array}$ & $\begin{array}{c}\tau_{0}^{\prime} \\
\text { (ns) }\end{array}$ \\
\hline \multicolumn{7}{|l|}{ Pyrene } \\
\hline \multirow[t]{5}{*}{ DBP } & $\begin{array}{l}\mathrm{MeCN} \\
\mathrm{MeCN}+\mathrm{O}_{2} \\
\mathrm{MeCN}+\mathrm{Q}^{\prime}\end{array}$ & 57 & 18 & $-4 \cdot 6$ & $\begin{array}{c}4 \pm 2 \\
6 \pm 2 \\
3 \pm 2\end{array}$ & $\begin{array}{r}17 \pm 5 \\
8 \pm 3 \\
8 \pm 3\end{array}$ \\
\hline & $\operatorname{PrCN}$ & $2 \cdot 6$ & 0.9 & 5.9 & $0.6 \pm 0.4$ & $35 \pm 20$ \\
\hline & AcOEt & 2.0 & 0.9 & $5 \cdot 7$ & & \\
\hline & $\mathrm{CH}_{2} \mathrm{Cl}_{2}$ & $7 \cdot 2$ & $2 \cdot 9$ & 3.8 & & \\
\hline & Toluene & $2 \cdot 3$ & 0.8 & $7 \cdot 1$ & & \\
\hline DEP & $\mathrm{MeCN}$ & 57 & 18 & $-6 \cdot 7$ & & \\
\hline DMB & $\mathrm{MeCN}$ & 14 & 4.7 & -7.0 & & \\
\hline $\mathrm{Et}_{2} \mathrm{NH}$ & $\mathrm{MeCN}$ & 99 & 33 & 10.5 & & \\
\hline $\mathrm{BuNH}_{2}$ & $\mathrm{MeCN}$ & $1 \cdot 2$ & 0.4 & $5 \cdot 0$ & & \\
\hline \multicolumn{7}{|c|}{ 9-Cyanoanthracene } \\
\hline DMN & $\mathrm{MeCN}$ & 80 & 490 & & 45 & 260 \\
\hline
\end{tabular}

Abbreviations: $\mathrm{DBP}=$ dibutylphthalate; $\mathrm{DEP}=$ diethylphthalate; $\mathrm{DMB}=1$,4-dimethoxybenzene; $\mathrm{Et}_{2}$. $\mathrm{NH}=$ diethylamine; $\mathrm{BuNH}_{2}=$ tert-butylamine; $\mathrm{Q}^{\prime}=$ dimethylfumarate; $\mathrm{DMN}=1,6$-dimethylnaphthalene; $\mathrm{MeCN}=$ acetonitrile; $\mathrm{PrCN}=$ butyronitrile; AcOEt = ethylacetate.

Formal activation energies $E_{F}^{+}$, calculated from the ratio of the diffusion rate constant in the solvent and apparent quenching rate constant at a given temperature (supposing that it is the activation enthalpy which is responsbile for their difference) are about $14-26$ and $20-25 \mathrm{~kJ} / \mathrm{mol}$ greater than the respective experimental ones.

\section{The nature of the exciplex}

The electronic structure of exciplexes is usually represented by a combination of the wavefunctions of locally excited and charge transfer states (if the excited state of the quencher can be neglected owing to much higher excitation energy):

$$
\Psi(\mathrm{AD})^{*}=a \Psi\left(\mathrm{A}^{*}\right) \Psi(\mathrm{D})+b \Psi\left(\mathrm{A}^{-} \mathrm{D}^{+}\right)
$$

Coefficients $a$ and $b$ depend on the difference of the energies of the locally excited and charge transfer states (which can be approximated by the enthalpy of an electron transfer $\Delta H_{\mathrm{ET}}$ ) and on the exchange interaction parameter $\beta$. Enthalpy of the exciplex $\Delta H_{\mathrm{Ex}}$ (neglecting the polarization of the solvent) and magnitude of electron transfer in the exciplex ${ }^{1}\left(\mathrm{M}^{-\delta} \mathrm{Q}^{+\delta}\right)^{*}$ depend on the same parameters:

$$
\begin{aligned}
\Delta H_{\mathrm{Ex}} & \approx \Delta H_{\mathrm{ET}} / 2-\left[\left(H_{\mathrm{ET}} / 2\right)^{2}+\beta^{2}\right]^{1 / 2}, \\
\delta & \approx 1 /\left\{1-\left(\Delta H_{\mathrm{ET}} / \beta\right) \cdot\left[\left(1+\left(\Delta H_{\mathrm{ET}} / 2 \beta\right)^{2}\right)^{1 / 2}-\Delta H_{\mathrm{ET}} / 2 \beta\right]\right\} .
\end{aligned}
$$

The enthalpy of exciplex formation will be negative not only for negative $\Delta H_{\mathrm{ET}}$ but 


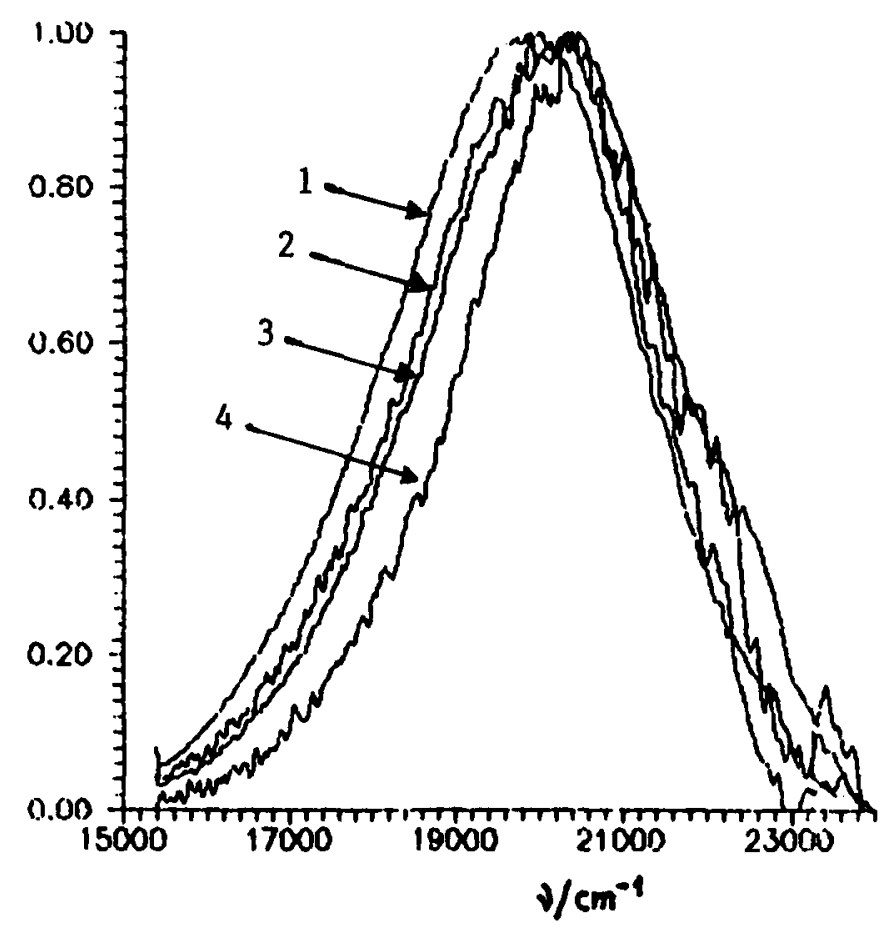

Figure 4. Corrected emission spectra of the exciplexes of 9-cyanoanthracene with 1,6dimethylnaphthalene in acetonitrile (1), butyronitrile (2), dichloromethane (3), and toluene (4).

also for $\Delta H_{\mathrm{ET}}$ close to zero and even positive $\Delta H_{\mathrm{ET}}$. For strongly negative $\Delta H_{\mathrm{ET}}$, $\delta \approx 1$ and ordinary polar exciplexes are formed whose structure is close to radical-ion pair. But for positive $\Delta H_{\mathrm{ET}}, \delta \ll 1$ and low polar exciplexes are formed (their electronic structure is similar to ordinary ground-state charge transfer complexes). According to (20) the smaller $\Delta H_{\mathrm{ET}}$, the greater the exciplex formation equilibrium constant $K_{\mathrm{Ex}}=\exp \left(-\Delta G_{\mathrm{Ex}} / R T\right)$. Emission maxima of the exciplex of 9-cyanoanthracene with 1,6-dimethylnaphthalene only slightly depends on the polarity of the solvent (figure 4) which confirms the low polarity of such exciplexes.

The emission bands of these low polar exciplexes can be very weak for several reasons: the low value of the equilibrium constant $K_{\mathrm{F}}$ and the low probability of radiative transition to the ground state. The emission band can also be masked by the main fluorescence band of pyrene.

Decay of the exciplex can also proceed by several ways: by internal conversion to the ground state (encounter complex $M \cdot Q$ ), by intersystem crossing to the triplet state (triplet exciplex) and by dissociation on free radical-ions (solvated). All these processes should have sufficiently slow rates $\left(<10^{8} \mathrm{~s}^{-1}\right)$ to provide the relatively long lifetime of the exciplex $\left(>10^{-8} \mathrm{~s}\right)$. For the latter process, this means that its activation energy is greater than $25 \mathrm{~kJ} / \mathrm{mol}$. Internal conversion and intersystem crossing can have low probability because of small values of Franck-Condon factor and spininversion factor respectively rather than because of high activation energy (which can be close to zero). All these four rate constants (including emission rate constant) should depend on the chemical nature of both fluorophore and quencher. 


\section{Simulation of the dependence of $k_{\mathrm{Q}}$ on $\Delta H_{\mathrm{ET}}$}

We simulated the dependence of $k_{\mathrm{Q}}$ on $\Delta H_{\mathrm{ET}}$ for both the kinetic models of fluorescence quenching, (1) and (6), using the following approximations.

Rate constants are expressed by the Arrhenius' equations

$$
k_{i j}=k_{i j}^{0} \cdot \exp \left(-E_{i j}^{\neq} / R T\right)
$$

Encounter complex formation in acetonitrile

$$
k_{12}^{0}=10^{11} \mathrm{M}^{-1} \mathrm{~S}^{-1} ; \quad E_{12}^{\neq}=5 \mathrm{~kJ} / \mathrm{mol}
$$

Equilibrium constant of encounter complex formation

$$
k_{12} / k_{21}=0.5 \mathrm{M}^{-1}, \Delta H_{12}=0 .
$$

For (1):

Electron transfer in the encounter complex

$$
\begin{aligned}
& k_{23}^{0}=10^{11} \mathrm{~s}^{-1} ; \quad \Delta G_{23}^{\neq}=\left(\Delta G_{\mathrm{ET}} / 2\right)+\left[\left(\Delta G_{\mathrm{ET}} / 2\right)^{2}+\left(\Delta G_{0}^{\neq}\right)^{2}\right]^{1 / 2}, \\
& \Delta G_{0}^{\neq}=10 \mathrm{~kJ} / \mathrm{mol} \text {. }
\end{aligned}
$$

Radical-ion pair decay

For (6):

$$
k_{30}=10^{11} \mathrm{~s}^{-1} ; \quad E_{30}^{\neq}=0
$$

Exciplex formation

$$
\begin{aligned}
k_{23} / k_{32} & =\exp \left(-\Delta H_{\mathrm{Ex}} / R T\right) ; & & k_{23}^{0}=10^{11} \mathrm{M}^{-1} \mathrm{~s}^{-1} \\
\Delta H_{\mathrm{Ex}} & \left.=\left(\Delta H_{\mathrm{ET}} / 2\right)-\left[\left(\Delta H_{\mathrm{ET}} / 2\right)^{2}+\beta^{2}\right)\right]^{1 / 2} ; & & \Delta H_{\mathrm{ET}} \approx \Delta G_{\mathrm{ET}} \\
E_{23}^{\neq} & =\left(\Delta H_{\mathrm{Ex}} / 2\right)+\left[\left(\Delta H_{\mathrm{Ex}} / 2\right)^{2}+\left(\Delta H_{0}^{+}\right)^{2}\right]^{1 / 2}, & & \Delta H_{0}^{\neq}=10 \mathrm{~kJ} / \mathrm{mol}
\end{aligned}
$$

$\beta$-variable parameter (initial value $\beta=E_{\mathrm{F}}^{\neq}-E_{\mathrm{Exp}}^{\neq}=-15 \mathrm{~kJ} / \mathrm{mol}$ ).

Exciplex decay

$$
\begin{aligned}
1 / \tau_{0}^{\prime} & =k_{30}^{0} \exp \left(-E_{30}^{\neq} / R T\right) \\
k_{30}^{0} & =A \text { and } E_{30}^{\neq}=E \text { are variable parameters. }
\end{aligned}
$$

For (6) apparent quenching rate constants $k_{\mathrm{Q}}$ were calculated from

$$
\begin{aligned}
k_{\mathrm{Q}}= & 10^{11} \exp (-5 / R T) /\left\{1+2 \times 10^{11} \exp (-5 / R T) /\left(10^{11} \exp \left(-E_{23}^{*} / R T\right)\right)\right. \\
& \left.+2 \times 10^{11} \exp (-5 / R T) \cdot \exp \left(\Delta G_{\mathrm{Ex}} / R T\right) /(A \cdot \exp (-E / R T))\right\} .
\end{aligned}
$$

Simulated dependence of $\ln k_{\mathrm{Q}}$ on $\Delta G_{\mathrm{ET}}$ according to the classical scheme (1) (curve 1) and the exciplex scheme (6) for various values of $\beta$ and $\tau_{0}^{\prime}$ (curves 2-9) are given in figure 5 . For $\Delta G_{\mathrm{ET}} \approx 0$, quenching rate constants according to (6) are smaller than those for (1). For $\Delta G_{\mathrm{ET}} \gg 0$, the quenching rate constants do not depend on $\Delta G_{\mathrm{ET}}$ and are greater than those expected according to the classical scheme (1). In this region of $\Delta G_{E T}$ values the quenching of excited molecules results from their 


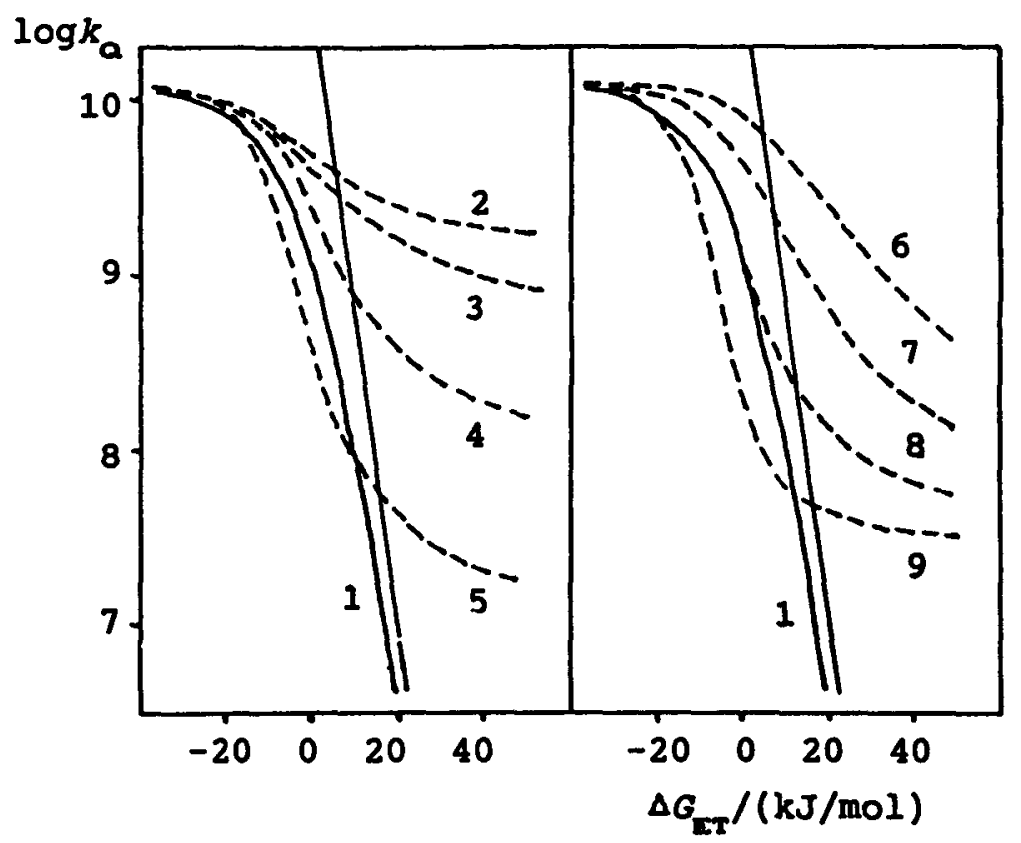

Figure 5. Plots of apparent fluorescence quenching rate constants $\left(\ln k_{\mathrm{Q}}\right)$ on Gibbs energy of electron transfer $\Delta G_{\mathrm{ET}}$ according to kinetic schemes (1) and (6): 1 - simulation according to scheme (1); 2-9 - simulation according to scheme (6). $2-5-\beta=10 \mathrm{~kJ} / \mathrm{mol}, \tau_{0}^{\prime}=0.01,1$, $10,100 \mathrm{~ns} ; 6-9-\tau_{0}^{\prime}=30 \mathrm{~ns}, \beta=20,15,10,5 \mathrm{~kJ} / \mathrm{mol}$.

conversion to the exciplex. A limiting value of the apparent quenching rate constant and the slope of the plot of $\log k_{\mathrm{Q}}$ vs. $\Delta G_{\mathrm{ET}}$ are functions of $\tau_{0}^{\prime}$ and $\beta$.

At fixed $\tau_{0}^{\prime}$ values, the rise of $\beta$ will result in the contraction of the range of the dependence of $k_{\mathrm{Q}}$ on $\Delta G_{\mathrm{ET}}$ and increase in the limiting value of $k_{\mathrm{Q}}$ (curves 2-4). At sufficiently large values of $\beta$, the quenching rate constant does not depend on $\Delta G_{E T}$ in the whole range $\Delta G_{\mathrm{ET}}>5 \mathrm{~kJ} / \mathrm{mol}$ because of the irreversibility of the exciplex formation. This exciplex decays by internal conversion and/or by intersystem crossing.

At fixed values of $\beta$, the decrease of the exciplex lifetime $\tau_{0}^{\prime}$ results in the increase of the quenching rate constant (curves 5-7). The greater the value of $\beta$, the greater the stability of the exciplex and the smaller the effect of $\tau_{0}^{\prime}$ on the apparent quenching rate constant. At sufficiently large values of $\beta$, the lifetime of the exciplex does not affect the quenching because of the irreversibility of the exciplex formation.

Activation energy of quenching can vary in wide range from negative to positive values in accordance with the parameters $\beta, \Delta G_{\mathrm{ET}}$ and $\tau_{0}^{\prime}$.

The exciplex scheme (6) represents quite well the experimental data for temperaturedependence of the apparent rate constant of pyrene fluorescence quenching by dibutylphthalate in acetonitrile at the following values of the parameters: $\beta=-13.5 \mathrm{~kJ} / \mathrm{mol}$, $A=1.1 \times 10^{9} \mathrm{~s}^{-1}, E=10 \mathrm{~kJ} / \mathrm{mol}, \Delta G_{\mathrm{ET}}=-2 \mathrm{~kJ} / \mathrm{mol}$ (figure 5). In butyronitrile the best fit of the experimental data was obtained at $\beta=-10 \mathrm{~kJ} / \mathrm{mol}, A=0.9 \times 10^{9} \mathrm{~s}^{-1}$, $E=11 \mathrm{~kJ} / \mathrm{mol}, \Delta G_{\mathrm{ET}}=+9 \mathrm{~kJ} / \mathrm{mol}$ (figure 5). At $20^{\circ} \mathrm{C} k_{30}$ is equal to $5 \times 10^{7} \mathrm{M}^{-1} \mathrm{~s}^{-1}$ in acetonitrile and $3 \times 10^{7} \mathrm{M}^{-1} \mathrm{~s}^{-1}$ in butyronitrile which are close to the values determined directly from fluorescence kinetics (table 1). In less polar butyronitrile 
the value of $\beta$ is slightly smaller than in acetonitrile. The values of activation energy and pre-exponential factor of exciplex decay are close in both solvents.

\section{Quenching dynamics}

Let us consider the nature of the potential barriers, along with the reaction coordinate in the course of fluorescence quenching, taking into account exchange interactions between reactants (coupling of locally excited and charge transfer states) in the frame of very simple approximations (figure 6).

For positive $\Delta G_{\mathrm{ET}}$ (figure 6 , top) as the reactants $\mathrm{D}^{*}$ and $\mathrm{A}$ approach each other, the energy decreases according with increase of the overlap of the wavefunctions of the reactants (increase of the exchange interaction energy $\beta$ ) and decrease of the energy $\left(E_{2}\right)$ of the charge transfer state $\left(\mathrm{D}^{+} \mathrm{A}^{-}\right)$.

$$
E \approx\left[\left(E_{1}+E_{2}\right) / 2\right]-\left[\left(E_{1}-E_{2}\right)^{2} / 4+\beta^{2}\right]^{1 / 2},
$$

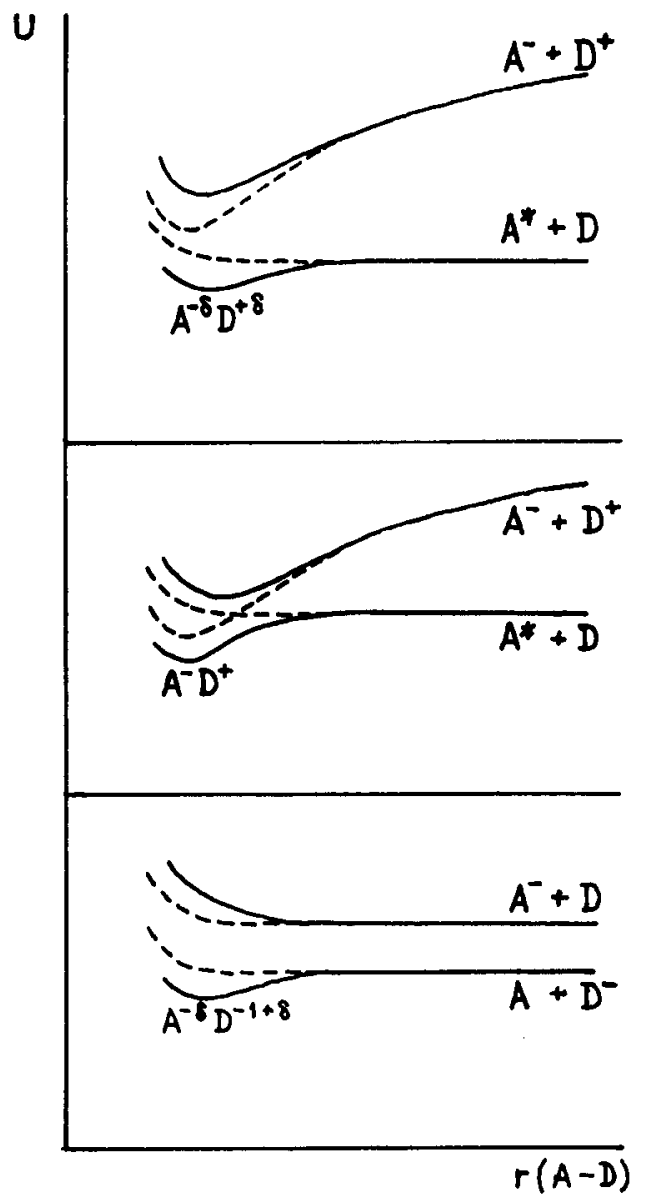

Figure 6. Potential energy curves for exciplex formation and electron transfer at $\Delta G_{\mathrm{ET}}>0$ (top) and at $\Delta G_{\mathrm{ET}}<0$ (middle) for uncharged reactants, and for an uncharged and a charged reactant (bottom). 
where $E_{1}$ is the energy of the locally excited state $\left(D^{*} A\right)$. An exciplex with a modest degree of electron transfer is formed. The degree of electron transfer increases gradually as reactants approach each other and solvent reorganization also gradually follows the polarization of the encounter complex.

Three potential barriers along with the reaction coordinate for the exciplex formation and subsequent complete electron transfer can arise. The first one is the diffusion barrier which has the usual activation energy in the range $5-10 \mathrm{~kJ} / \mathrm{mol}$. The second is the activation energy of exciplex formation which can be formally approximated by:

$$
E_{23}^{\neq}=\left(\Delta H_{\mathrm{Ex}} / 2\right)+\left[\left(\Delta H_{\mathrm{Ex}} / 2\right)^{2}+\left(\Delta H_{0}^{\ddagger}\right)^{2}\right]^{1 / 2}
$$

assuming $\Delta H_{0}^{\neq}=10 \mathrm{~kJ} / \mathrm{mol}$, similar to Weller's model (this value was found to be unessential for the results of the simulations since equilibrium between the exciplex and the reactants is established at $\Delta G_{\mathrm{ET}}>0$ ).

Complete electron transfer in this exciplex needs activation energy of about $\left[\Delta H_{\mathrm{ET}}^{2}+4 \beta^{2}\right]^{1 / 2}$ and yields a contact radical-ion pair (which can dissociate on free (solvated) radical-ions). Therefore, dominant ways of decay of the exciplex formed are internal conversion, intersystem crossing and emission (which do not need substantial activation energy), rather than complete electron transfer and formation of radical-ions. The rates of the first two radiationless decay processes may be very responsive to the chemical nature of the reactants. Therefore the lifetime of the exciplex can vary in the wide range $\sim 10^{-12}-10^{-7} \mathrm{~s}$ and may depend on the chemical nature of both excited molecule and quencher.

For negative $\Delta G_{\mathrm{ET}}$ (middle part of figure 6), the approach of the reactants towards each other can also be followed by the gradual increase of the charge separation but (in contrast to $\Delta G_{\mathrm{ET}}>0$ ) it finally produces a radical-ion state. This is the most well-known kind of exciplex. Along with reaction coordinates, the exchange interaction and the magnitude of charge transfer increase gradually. The solvent reorganization also occurs gradually and reorganization energy is much smaller than is expected from the Marcus theory.

Completely different nature of the activation barrier can be expected for electron transfer charged and uncharged molecules (figure 6, bottom). In this case, electrostatic interaction does not exist in either the initial or the final states and the potential curves of both the states in the first approximation are parallel to each other. for exergonic $\left(\Delta G_{\mathrm{ET}}<0\right)$ electron transfer the inclusion of exchange interactions will produce the repulsion of the potential curves (the rise of the energy of the initial state $D^{*}+A$ which can be expressed by the analog of (21) but with the positive sign ahead of the square root). This rise of energy will depend on the distance of electron transfer and will build up an additional potential barrier for electron transfer. Therefore, some additional contribution to the activation energy of electron transfer due to an exchange interaction between reactants must be taken into account in the models of electron transfer processes at negative $\Delta G_{\mathbf{E T}}$.

\section{Conclusions}

The model assuming the formation of sufficiently long-lived (up to 10-50 ns) exciplexes even in polar solvents is able to explain and quantitatively describe the experimentally observed abnormal temperature effect and lifetime dependence of fluorescence quenching 
in polar solvents and also the deviations of the experimental dependence of the quenching rate constants on $\Delta G_{\mathrm{ET}}$ from that predicted by the electron transfer theory of the quenching (especially in the kinetic region). The stabilization energy of the exciplex at positive $\Delta G_{\mathrm{ET}}$ can arise from exchange interactions between reactants (similar to ground state CT complexes); such exciplexes may have a low contribution of charge transfer state. The radiationless decay of the exciplex does not need any activation energy. This mechanism does not suppose the necessity of complete electron transfer between reactants in the excited state. Therefore, fluorescence quenching cannot be used with confidence for the verification of the theories of electron-transfer processes and for the determination of the redox-potentials of quenchers.

The exciplex model of quenching supposes that there are two main parameters exciplex formation equilibrium constant $K_{\mathrm{Ex}}$ and its lifetime $\tau_{0}^{\prime}-$ which determine the apparent value of the fluorescence quenching rate constant. The abnormal temperature effect arises from the negative enthalpy of the exciplex formation (in contrast to the positive activation energy of electron transfer).

The correlation between quenching rate constants and the free energy of electron transfer $\Delta G_{\mathrm{ET}}$ has different origins in the classical Weller's model, (1), and in the exciplex model, (2). In the classical model, two parameters which control the value of the quenching rate constant are the activation energy of isoergonic reaction $\Delta G_{0}^{*}$ and the lifetime of the radical-ion pair $\left(1 / k_{30}\right)$. The last one was supposed to be $0.01 \mathrm{~ns}$ and $\Delta G_{0}^{*}$ was found to be $10 \mathrm{~kJ} / \mathrm{mol}$ (Rehm and Weller 1970). The last one, $\Delta G_{0}^{\ddagger}$, was assumed to be variable to explain the variations in the $\log k_{\mathrm{Q}} \mathrm{vs} . \Delta G_{\mathrm{ET}}$ relationships.

In the exciplex model (6), the dependence of apparent quenching rate constants on $\Delta G_{\mathrm{ET}}$ arises from the dependence of the exciplex formation enthalpy on $\Delta G_{\mathrm{ET}}$, (20). The lifetime of the exciplex may exceed by many orders of magnitude the lifetime of radical-ion pairs in polar solvents and can vary in a very wide range, depending on the chemical structure of both the excited molecule and quencher. Positive and negative deviations from classical Weller's curve $\log k_{\mathrm{Q}}$ vs. $\Delta G_{\mathrm{ET}}$ may arise from the variations of the dependence of $K_{\mathrm{Ex}}$ on $\Delta G_{\mathrm{ET}}$ and from the variations of $\tau_{0}^{\prime}$. In consistence with numerous experimental data the slope of $\log k_{\mathrm{Q}} \mathrm{vs.} \Delta G_{\mathrm{ET}}$ plot in the kinetic region in the frame of the exciplex model (6) as a rule is much smaller than for thermodynamic limit (which is $5.9 \mathrm{~kJ}$ per one logarithmic unit).

\section{References}

Avila V, Cosa J J, Chesta C A and Previtali C M 1991 J. Photochem. Photobiol. A62 83

Baggott J E and Pilling M J 1983 J. Chem. Soc., Faraday Trans. 79221

Carrera A, Cosa J J and Previtali C M 1991 J. Photochem. Photobiol. A56 267

Fox M A and Chanon M (eds) 1988 Photoinduced electron transfer (Amsterdam: Elsevier) vol. A-D

Gordon M and Ware W R (eds) 1975 The exciplex (New York: Academic Press)

Hishimura T, Nakashima N and Mataga N 1977 Chem. Phys. Lett. 46334

Kitamura N, Obata R, Kim H -B and Tazuka S 1987 J. Phys. Chem. 912033

Kuzmin M G, Sadovskii N A, Weinstein J A and Soloveichik O M 1992 High Energy Chem. 26522 (in Russian)

Kuzmin M G and Soboleva I V 1986 Prog. React. Kinet. 14157

Neumann M G and Pastre I A 1991 J. Photochem. Photobiol 6191

Marcus R A 1956 J. Chem. Phys. 24966

Rehm D and Weller A 1969 Ber. Bunsenges Phys. Chem. 73834

Rehm D and Weller A 1970 Israel J. Chem. 8259 